\title{
Child abuse and the prevalence of suicide attempts among those reporting suicide ideation
}

\author{
Michael S. Martin ${ }^{1}$ Jennifer Dykxhoorn ${ }^{1,2} \cdot$ Tracie O. Afifi $^{3} \cdot \operatorname{Ian~Colman~}^{1}$
}

Received: 15 January 2016/Accepted: 5 June 2016/Published online: 11 June 2016

(C) Springer-Verlag Berlin Heidelberg 2016

\begin{abstract}
Objective Victims of child abuse may be at increased risk of acting on suicide ideation, although this has not been empirically tested. We estimated the risk of suicide attempts associated with child abuse among individuals who reported suicide ideation.

Methods Secondary analysis of data from the populationbased Canadian Community Health Survey Mental Health $(n=828)$. This population-based survey included various structured questionnaires, including the Composite International Diagnostic Interview to assess mental illness and suicidal thoughts and behaviours.

Results Approximately $80 \%$ of those who attempted suicide had a history of child abuse. Poor mental health, financial difficulties, poor coping skills, and reporting a suicide plan were also associated with an increased prevalence of attempting suicide; adjusted for these factors, child abuse was associated with a 1.77 -fold increased prevalence $(95 \%$ CI $0.93,3.36)$ of suicide attempts.

Conclusions Most individuals who attempt suicide experience child abuse, and worse health and social functioning.
\end{abstract}

Electronic supplementary material The online version of this article (doi:10.1007/s00127-016-1250-3) contains supplementary material, which is available to authorized users.

Michael S. Martin

mmart007@uottawa.ca

1 School of Epidemiology, Public Health and Preventive Medicine, University of Ottawa, 451 Smyth Road, Office 3230A, Ottawa, ON K1H8M5, Canada

2 Division of Psychiatry, University College London, London, UK

3 Departments of Community Health Sciences and Psychiatry, University of Manitoba, Winnipeg, Canada
Adopting a life-course perspective to understand trajectories of suicide risk factors may inform prevention and treatment.

Keywords Suicide $\cdot$ Child abuse $\cdot$ Mental disorders $\cdot$ Risk factors

\section{Introduction}

As suicide is one of the top 20 causes of death worldwide [1], there has been substantial effort to understand, predict, and prevent it. Klonsky and May [2] argue that research on risk for suicide has often conflated why people feel suicidal with why they act on suicidal thoughts. In a large international study of almost 85,000 individuals in 17 countries [3], approximately one-third of individuals reporting suicide ideation had a suicide attempt. For clinicians, who are most likely to encounter individuals who are already experiencing suicide ideation, it is most relevant to understand factors that predict (to inform risk assessment) or explain (to inform risk management) who will act on these thoughts. Many studies report higher rates of suicide ideation and attempts among those who experienced child abuse [4-10]. However, none have examined whether it is a risk factor for acting on suicide ideation. These studies may, therefore, be uninformative to clinicians, as many predictors of suicide ideation do not predict who will attempt suicide [2, 11, 12].

Various theories suggest that individuals who experience child abuse are at increased risk of accumulating additional risk factors for suicide, and that they more likely to act on thoughts of suicide ideation [11, 13, 14]. For example, Joiner's interpersonal-psychological theory of suicide posits that the progression from suicide ideation to 
an attempt requires an acquired capacity for suicide, which "results from exposure and attendant habituation to the pain and fear involved in physical self-harm" [14] (p. 643). Empirical tests of acquired capacity for suicide has typically been operationalized in terms of prior suicide attempts and non-suicidal self-harm. However, it is also postulated that experiencing painful and fear-inducing behaviours, including being a victim of abuse or witnessing others' pain and injury can increase capacity for suicide [14, 15], suggesting an independent association between child abuse and suicide attempts.

Few other predictors of suicide attempts among suicide ideators have been identified. Only the presence of a suicide plan $[3,12]$ and a history of self-harm or suicide have consistently been shown to predict the progression from suicide ideation to an attempt [3, 14, 16, 17]. Factors, such as mental illness [3, 12, 14, 18-21], substance abuse [3, 12, 19], sex [3, 12, 20-22], age [3, 12, 21, 22], marital status [3, 19], social interactions and belonging [14, 19], and life stressors [14, 18] have had conflicting findings. Other potential risk factors, such as financial strain [23, 24] and physical illness [25, 26], have not been studied as factors that may distinguish suicide ideators and attempters.

\section{Aims of the study}

Our primary objective was to test the association between child abuse and past year suicide attempts among individuals who reported suicide ideation in the past year. We focused only on past year outcomes to maintain the temporal order of childhood abuse and adult health and social functioning, including suicide thoughts and attempts, and to examine the long-term outcomes of individuals experiencing child abuse. Our secondary objectives were to identify proximal health and social outcomes associated with suicide attempts, and to assess whether these factors: (1) attenuated the association between child abuse and suicide attempts or (2) were differentially associated with suicide attempts depending on the presence of child abuse.

\section{Methods}

\section{Participants}

Data collected by Statistics Canada in 2012 as part of the nationally representative Canadian Community Health Survey-Mental Health [27] were analyzed for the current study. This study is a nationally representative survey of the Canadian population, excluding the three territories, Aboriginal reserves or other settlements, active military

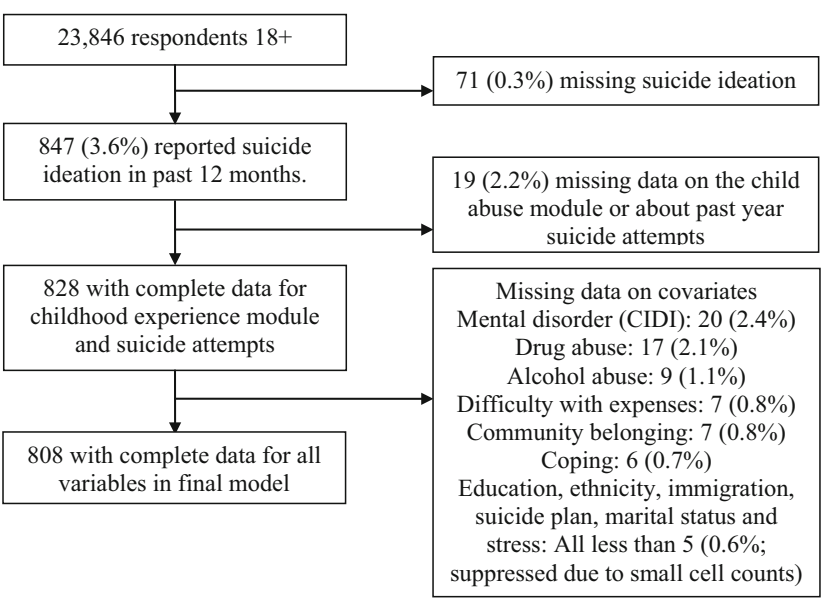

Fig. 1 Participant flow-diagram

members, and institutionalized populations (who account for less than $3 \%$ of the total population) [27]. Interviews were conducted primarily in person (89\%), although a small number were conducted by phone (11\%). 23,846 Canadians aged 18 years and older were asked questions regarding child abuse. After restricting our sample to those reporting suicide ideation with complete data on the primary exposure and outcome (see Fig. 1), our final sample included 828 respondents who reported suicide ideation in the past year (3.6\% of the original sample). Fewer than $5 \%$ of participants were missing data on all proximal health and social variables [ranging from no missing data to a maximum of $20(2.4 \%)$ missing per variable; see Fig. 1]; consequently, listwise deletion was used when these variables were included in adjusted models. Full details of the sampling allocation by province, three-stage sampling strategy (i.e., random selection of geographic areas, followed by households within those areas, and finally, a single respondent within each household), and the derivation of weights are provided in the Survey User Guide [27].

\section{Measures}

Child abuse Child abuse was measured using items from the Childhood Experiences of Violence Questionnaire (CEVQ) [28]. This questionnaire includes six items regarding childhood experiences that are answered using a 4 point scale to indicate how often the events occurred ranging from: never; once or twice; 3-5, 6-10, or 10 or more times. Following the recommendations of the authors of the CEVQ, we used a binary classification of experiencing child abuse or not. Participants were considered to have experienced child abuse if any of the following conditions were met before the age of 15: (1) slapped on the face, head or ears, or hit or spanked with something hard at 
least three times by an adult; (2) pushed, grabbed, shoved, or having something thrown at them at least three times by an adult; (3) witnessing or hearing parents or guardians hit each other or another adult in the house at least three times; (4) kicked, bit, punched, choked, burned, or physically attacked at least once by an adult; (5) forced (including attempts) into unwanted sexual activity by threat or physical restraint at least once by an adult; (6) unwanted touching or grabbing, or kissing or fondling against the respondent's will at least once by an adult. In secondary analyses, we disaggregated abuse types by physical abuse (experiences 1,2, and 4), sexual abuse (experiences 5 and 6 ), and witnessing family violence (experience 3 ). The CEVQ has high test-retest reliability, and agreement with clinician ratings of childhood victimization (i.e., kappa's of 0.5 and greater) [28].

Suicide thoughts and attempts Suicide ideation, suicide plans, and suicide attempts were assessed by three questions from the Composite International Diagnostic Interview (CIDI). Participants were asked if three experiences had happened to them ever, and if yes, in the past 12 months: (1) "you seriously thought about committing suicide or taking your own life?"; (2) "you made a plan for committing suicide?"; and (3) "you attempted suicide or tried to take your life?". Participants were only asked the 12 month questions if they reported the experience at any point in their lifetimes. For participants who answered 'No' to the lifetime suicide attempt item, we recoded 'Not applicable' responses to the 12 month timeframe items to 'No'. Participants who reported never having thought about suicide were not asked if they had made a plan or an attempt.
Demographic, health and social variables Demographic variables (e.g., sex, age, and ethnicity) and recent health and social functioning variables were included in models to assess whether associations between child adversity and adult suicide attempts were independent of these variables that reflected the individuals' circumstances closer in time to the experience of suicide ideation. Past 12 month diagnoses of major depressive episode, bipolar disorder, generalized anxiety disorder, and drug or alcohol abuse or dependence were made using the CIDI, which has been used in large studies of mental health and suicide, and shown to have high reliability and validity [3, 29]. Because there was no module for psychotic disorders, we used participant self-report of a diagnosis of schizophrenia or any other psychosis from a health professional that is "expected to last or has already lasted 6 months or more". Other measures of health and social functioning in the past year have been developed by Statistics Canada as part of annual health surveys to minimize response burden and increase participation rates, in light of the range of topics addressed. While we are unaware of established psychometric properties of these items, they are face valid, and those which have been compared against longer assessment instruments have been shown to measure similar constructs [30]. To comply with Statistics Canada policies to protect respondent confidentiality (i.e., cross tabulations with fewer than ten respondents in each cell cannot be released), we collapsed these variables to indicate whether respondents reported: poor mental health, poor physical health, high stress, poor ability to cope with daily stressors, poor ability to cope with unexpected stressors, and a strong sense of belonging to a community (see Table 1 for the

Table 1 Survey questions and coding in analyses

\begin{tabular}{|c|c|c|c|}
\hline Variable & Question & Options to respondent & Coding \\
\hline Perceived physical health & In general, would you say your health is... & $\begin{array}{l}\text { Excellent, very good, good, } \\
\text { fair, poor }\end{array}$ & Poor vs others \\
\hline Perceived mental health & In general, would you say your mental health is... & $\begin{array}{l}\text { Excellent, very good, good, } \\
\text { fair, poor }\end{array}$ & Poor vs others \\
\hline Life stress & $\begin{array}{l}\text { Thinking about the amount of stress in your life, } \\
\text { would you say most days are... }\end{array}$ & $\begin{array}{l}\text { Not at all stressful, not very } \\
\text { stressful, a bit stressful, } \\
\text { quite a bit stressful, } \\
\text { extremely stressful }\end{array}$ & $\begin{array}{l}\text { Quite a bit and extremely } \\
\text { stressful vs all others }\end{array}$ \\
\hline Community belonging & $\begin{array}{l}\text { How would you describe your sense of belonging } \\
\text { to your local community? Would you say it is... }\end{array}$ & $\begin{array}{l}\text { Very strong, somewhat } \\
\text { strong, somewhat weak, } \\
\text { very weak }\end{array}$ & Very strong vs others \\
\hline General coping & $\begin{array}{l}\text { In general, how would you rate your ability to } \\
\text { handle the day-to-day demands in your life, for } \\
\text { example, handling work, family, and volunteer } \\
\text { responsibilities }\end{array}$ & $\begin{array}{l}\text { Excellent, very good, good, } \\
\text { fair, poor }\end{array}$ & Poor vs others \\
\hline Coping with problems & $\begin{array}{l}\text { In general, how would you rate your ability to } \\
\text { handle unexpected and difficult problems, for } \\
\text { example, a family or personal crisis }\end{array}$ & $\begin{array}{l}\text { Excellent, very good, good, } \\
\text { fair, poor }\end{array}$ & Poor vs others \\
\hline
\end{tabular}


exact wording of these questions, and re-coding of responses for analyses).

\section{Statistical analysis}

We calculated the relative risk of the likelihood that respondents who reported child abuse also reported a suicide attempt in the past 12 months. We used the procedure proposed by Zou to estimate relative risk for a common (i.e., prevalence of $10 \%$ or more) dichotomous outcome using Poisson regression with a robust variance estimator [31]. To address our secondary objectives, we calculated unadjusted relative risks for more recent health and social functioning outcomes which are associated with child abuse to test whether they also predicted suicide attempts. We then calculated adjusted relative risks for child abuse controlling for each of these risk factors to assess whether any of these factors could account for the association. Finally, we tested whether there was an interaction between child abuse and each of these proximal risk factors. Sampling weights to account for the complex sampling procedures were used in all analyses.

\section{Ethics statement}

Ethics approval was provided by the Ottawa Health Science Network Research Ethics Board (protocol number 20140183-01H). As we conducted secondary analysis of de-identified data collected by Statistics Canada, we were not involved in the informed consent process. Informed consent was obtained by Statistics Canada Interviewers prior to commencing the questionnaire.

\section{Results}

As seen in Table 2, participants who reported suicide ideation in the past 12 months reported high rates of child abuse $(61.2 \%)$ as well as a range of poor health and social outcomes. Overall, $14.3 \%$ of participants who reported suicide ideation in the past 12 months attempted suicide during this same time period. However, the prevalence of suicide attempts was 2.56 times (95\% CI 1.29, 5.08) higher among those reporting child abuse (18.7\%) than among those not reporting child abuse (7.3\%). The majority $(80.1 \%)$ of those with a suicide attempt reported child abuse. Supplementary analyses disaggregating child abuse by sub-type found a stronger association between sexual abuse $(\mathrm{RR}=2.65,95 \%$ CI 1.36, 5.14) than for physical abuse or witnessing violence (RR's of 1.60 and 1.73 , respectively, with confidence intervals crossing 1 ; see Table 3).
Table 2 Weighted prevalence of suicide attempts and characteristics of interest among individuals reporting suicide ideation

\begin{tabular}{lr}
\hline Variable & $\%$ \\
\hline Child abuse & 61.2 \\
Physical abuse & 49.3 \\
Sexual abuse & 30.5 \\
Witness family violence & 26.2 \\
Suicide attempt & 14.3 \\
Suicide plan & 31.3 \\
Mental disorder & 52.7 \\
Major depressive episode & 42.4 \\
Bipolar & 12.1 \\
Generalized anxiety disorder & 21.3 \\
Psychosis & 8.7 \\
Number of mental disorders & \\
One mental disorder & 29.4 \\
2+ disorders & 23.3 \\
Alcohol abuse/dependence & 12.0 \\
Drug abuse/dependence & 9.3 \\
Poor mental health & 15.1 \\
Poor physical health & 11.0 \\
Female & 51.6 \\
Non-white & 24.0 \\
Aboriginal & 10.0 \\
Immigrant & 17.2 \\
Did not finish high school & 20.2 \\
Unemployed & 31.7 \\
Lowest income quintile & 33.7 \\
Difficulty meeting expenses & 31.1 \\
Married/common law & 45.2 \\
Strong community belonging & 43.5 \\
High stress & 47.2 \\
Poor coping (unexpected events) & 12.2 \\
Poor daily coping & \\
\hline &
\end{tabular}

As seen in the first column of Table 3, few of the demographic and recent health and social functioning variables were associated with suicide attempts. Having a suicide plan, mental illness (specifically major depressive and psychotic disorders), being in the lowest income quintile, having difficulty meeting basic expenses, and having poor coping skills to handle unexpected events were associated with a higher prevalence of suicide attempts. Other factors, such as drug and alcohol abuse or dependence, perceived stress, marital status, unemployment, community belonging, and poor coping with daily events were not significantly associated with suicide attempts. After adjustment for each of the significant variables individually, the association between child abuse and suicide attempts remained significant, and with an adjusted 
Table 3 Relative risks [95\% CIs] of suicide attempts among those reporting suicide ideation

\begin{tabular}{|c|c|c|c|c|c|}
\hline & \multirow[t]{2}{*}{ RR for risk factor } & \multicolumn{4}{|c|}{ RR of child abuse predicting suicide attempt, adjusted for risk factor } \\
\hline & & Any & Physical & Sexual & Witness violence \\
\hline Child abuse (unadjusted) & & $2.56[1.29,5.08]$ & $1.60[0.67,3.81]$ & $2.65[1.36,5.14]$ & $1.73[0.83,3.60]$ \\
\hline Suicide plan & $5.33[2.49,11.39]$ & $2.09[1.08,4.07]$ & $1.44[0.64,3.25]$ & $2.26[1.24,4.13]$ & $1.75[0.85,3.61]$ \\
\hline Any SMI & $3.91[1.90,9.07]$ & $2.19[1.15,4.18]$ & $1.35[0.58,3.16]$ & $2.07[1.14,3.78]$ & $1.71[0.87,3.35]$ \\
\hline MDE & $4.03[2.11,7.69]$ & $2.13[1.13,4.02]$ & $1.35[0.58,3.12]$ & $2.06[1.15,3.70]$ & $1.74[0.90,3.35]$ \\
\hline Bipolar & $1.52[0.59,3.96]$ & $2.40[1.19,4.85]$ & $1.47[0.63,3.42]$ & $2.67[1.32,5.37]$ & $1.67[0.81,3.45]$ \\
\hline GAD & $1.03[0.54,1.95]$ & $2.56[1.27,5.14]$ & $1.61[0.68,3.77]$ & $2.59[1.29,5.21]$ & $1.70[0.81,3.56]$ \\
\hline Psychosis & $3.00[1.40,6.44]$ & $2.39[1.19,4.80]$ & $1.42[0.62,3.27]$ & $2.34[1.12,4.87]$ & $1.51[0.77,2.95]$ \\
\hline Number of SMI & & $2.21[1.14,4.28]$ & $1.36[0.62,2.97]$ & $2.09[1.11,3.93]$ & $1.72[0.92,3.23]$ \\
\hline One MD & $3.89[1.62,9.30]$ & & & & \\
\hline $2+\mathrm{MD}$ & $3.95[1.85,8.43]$ & & & & \\
\hline Alcohol abuse/dependence & $0.93[0.46,1.88]$ & $2.62[1.31,5.25]$ & $1.65[0.69,3.92]$ & $2.63[1.36,5.09]$ & $1.76[0.85,3.66]$ \\
\hline Drug abuse/dependence & $0.81[0.37,1.81]$ & $2.54[1.24,5.20]$ & $1.54[0.64,3.74]$ & $2.78[1.40,5.53]$ & $1.71[0.80,3.70]$ \\
\hline Poor mental health & $3.01[1.36,6.66]$ & $2.28[1.20,4.34]$ & $1.67[0.79,3.55]$ & $2.45[1.38,4.37]$ & $1.78[0.86,3.65]$ \\
\hline Poor physical health & $1.39[0.67,2.90]$ & $2.55[1.28,5.07]$ & $1.58[0.67,3.74]$ & $2.62[1.33,5.18]$ & $1.74[0.83,3.63]$ \\
\hline Female & $1.34[0.67,2.70]$ & $2.53[1.30,4.95]$ & $1.62[0.70,3.76]$ & $2.60[1.40,4.83]$ & $1.71[0.82,3.57]$ \\
\hline Non-white & $1.77[0.85,3.70]$ & $2.43[1.20,4.96]$ & $1.46[0.61,3.53]$ & $2.48[1.17,5.26]$ & $1.39[0.68,2.82]$ \\
\hline Aboriginal & $\mathrm{n}<10$ could not $\mathrm{r} e$ & & & & \\
\hline Immigrant & $1.74[0.84,3.59]$ & $2.60[1.33,5.09]$ & $1.64[0.67,4.05]$ & $2.81[1.47,5.35]$ & $1.66[0.80,3.43]$ \\
\hline Did not finish high school & $1.46[0.67,3.14]$ & $2.49[1.25,4.97]$ & $1.55[0.66,3.61]$ & $2.64[1.36,5.12]$ & $1.66[0.83,3.33]$ \\
\hline Unemployed & $1.39[0.67,2.87]$ & $2.60[1.29,5.25]$ & $1.57[0.65,3.77]$ & $2.56[1.25,5.27]$ & $1.61[0.82,3.16]$ \\
\hline Lowest income quintile & $3.33[1.74,6.34]$ & $2.10[1.12,3.95]$ & $1.41[0.61,3.25]$ & $2.09[1.17,3.74]$ & $1.43[0.69,2.99]$ \\
\hline Difficulty meeting expenses & $2.21[1.09,4.47]$ & $2.28[1.21,4.26]$ & $1.48[0.61,3.57]$ & $2.35[1.32,4.19]$ & $1.56[0.73,3.32]$ \\
\hline Married/common law & $0.60[0.28,1.28]$ & $2.58[1.31,5.10]$ & $1.59[0.68,3.74]$ & $2.59[1.36,4.93]$ & $1.88[0.95,3.72]$ \\
\hline Strong community belonging & $1.12[0.52,2.45]$ & $2.60[1.29,5.21]$ & $1.63[0.76,3.54]$ & $2.69[1.36,5.34]$ & $1.73[0.87,3.44]$ \\
\hline High stress & $1.45[0.72,2.91]$ & $2.47[1.27,4.81]$ & $1.58[0.67,3.75]$ & $2.57[1.36,4.84]$ & $1.64[0.75,3.63]$ \\
\hline Poor coping (unexpected events) & $2.80[1.39,5.64]$ & $2.41[1.21,4.83]$ & $1.42[0.62,3.25]$ & $2.40[1.18,4.89]$ & $1.59[0.82,3.09]$ \\
\hline Poor daily coping & $1.52[0.67,3.43]$ & $2.57[1.30,5.10]$ & $1.58[0.66,3.75]$ & $2.67[1.38,5.17]$ & $1.72[0.82,3.59]$ \\
\hline
\end{tabular}

Bold values represent relative risks that are statistically significant at $\alpha=0.05$

relative risk ranging between 2.09 and 2.42. After adjusting for all of the recent health and social functioning variables, the relative risk of suicide attempts among those reporting child abuse was attenuated by approximately half $(\mathrm{RR}=1.77,95 \% \mathrm{CI} 0.93,3.36)$. The patterns of results are similar for each type of abuse, where controlling for individual predictors had a minimal impact on the unadjusted relative risk associated with child abuse (see the last 3 columns of Table 3 ).

Finally, we tested for effect modification to determine whether the association between each of the proximal factors identified in Table 3 and suicide attempts differed among those who reported child abuse compared to those who did not. None of these interactions were statistically significant at an alpha of 0.05 ( $p$ values were all 0.2 or larger for these interaction terms). Only the interaction term between child abuse and a mental health issue suggested a trend towards a stronger association among those who reported child abuse $(p=0.097)$.

\section{Discussion}

In this large study of individuals who report suicide ideation, we found that approximately four in five individuals with suicide attempts in the past year reported child abuse. Clinically, understanding the characteristics that distinguish those who think about suicide from those who attempt suicide is critical for determining how best to respond to an individual reporting with suicide ideation. While unadjusted results suggested risk of suicide attempts was more than twice as high among individuals with a history of child abuse, after adjusting for the four more recent indices of health and social functioning that were associated with suicide attempts (e.g., presence of a suicide plan, poor mental health, financial difficulties, and poor coping skills), this association was approximately half of its unadjusted value (attenuated from 2.56 to 1.77). As these factors are all associated with child abuse, longitudinal research is needed to clarify whether these proximal 
factors mediate or confound the association between childhood adversity and suicide behaviour. Our findings suggest a stronger association between childhood sexual abuse than physical abuse or witnessing violence. These findings are consistent with other studies on child abuse and suicide [9, 32], although given wide confidence intervals, risk of suicide among victims of physical abuse, or among those who witnessed family violence may warrant further study, given that both types of abuse were associated with a $75 \%$ increase in risk among our study sample. We did not find evidence that proximal social and health variables were more strongly associated with suicide attempts among individuals who reported child abuse, as suggested by the diathesis-stress model [13, 33-35]. These findings suggest that while child abuse is associated with increased likelihood of thinking about suicide (as shown by numerous past studies [4-6, 9]), current health, and social functioning must be considered when considering the higher prevalence of suicide attempts among people with a history of child abuse.

Our findings are consistent with past studies that have shown strong associations between the presence of a suicide plan and suicide attempts $[3,12]$. While we are unaware of other studies investigating financial strain as a characteristic distinguishing suicide ideators and attempters, previous work has found that financial stressors may contribute to a substantial proportion of suicide attempts $[23,36]$. Findings related to mental disorder and self-rated mental health are consistent with some population-based studies [19] that report increased risk of suicide attempts among individuals with suicide ideation and diagnoses of depression in particular. However, they are inconsistent with findings of Nock and colleagues, who reported that while all categories of disorders were associated with a higher prevalence of suicide attempts, this risk was restricted to those with three or more disorders [3]. It is unclear why some categories of disorders-e.g., anxiety and substance use disorders-were not related with suicide attempts, nor why we observed an association for individuals whether they had one or multiple diagnoses. Further work is needed to clarify the association between mental disorder and suicide attempts among suicide ideators.

Given that no single variable could fully account for the observed association between child abuse and suicide attempts, our results suggest that it is accumulation of risk factors that may be important to understanding the overrepresentation of child abuse among those who attempt suicide. While few studies have considered combinations of risk factors, others have suggested that an accumulation of risk appears to be particularly relevant $[3,37]$. These findings are consistent with perspectives, such as O'Connor's integrated motivational-volitional theory of suicide behaviour [38], which suggests that background events, such as child abuse, followed by multiple various intermediate variables increase motivation and volition for suicide.

While our study is among the largest studies to examine the progression from suicide ideation to attempts in a general population survey with a high response rate, little missing data and using well-validated measures for primary variables, such as child abuse, mental health, and suicide, its limitations should also be mentioned. First, the cross-sectional nature of the data introduces the possibility of recall bias. This may be of particular concern for the recall of child abuse. Prior research considering a broader range of adverse experiences than child abuse, including hospitalizations, parental substance abuse and unemployment, and frightening events that were thought about years after, has shown inconsistent reporting when the same questions were asked 12 years apart [39]. The cross-sectional design also precluded examining the time ordering of events. As a result, we excluded a number of potentially important characteristics that may reduce risk of suicide, such as receipt of mental health services and physical activity, due to the potential for these variables to be outcomes that followed suicidal thoughts or attempts. This was either due to the fact that some items, such as physical activity, were assessed over a more recent (i.e., shorter window), such as the past week, or due to the fact that questions were not asked to distinguish mental health services prior to or following suicidal thoughts or attempts. A final design limitation is the reliance on un-validated single items to capture complex variables (e.g., stress rather than specific life events). While this limits our ability to speak about the use of specific events as predictors of risk, stress sensitization hypotheses + that stress appraisals and tolerance may be more important in the development of adverse mental health outcomes than the stressor itself [13].

A second limitation pertains to the skip patterns established as part of the survey, which require the presence of suicide ideation to proceed to questions regarding a suicide plan and attempt. While these skip patterns fit the definition of suicide-(i.e., a suicide attempt is distinguished from non-suicidal behaviour based on an intent to die, thus requiring the presence of thoughts of suicide)—it is unknown whether respondents' descriptions of their suicidal thoughts and behaviours are consistent with this operationalization of these outcomes. Thus, we cannot comment on the prevalence of individuals who do not proceed along a linear continuum of suicidal thoughts and behaviours (i.e., from thoughts to attempts) or their characteristics. Given the lack of attention to this question in the literature about the measurement of suicide ideation and attempts, and the common use of these types of skip 
patterns (i.e., the use of the CIDI in other large studies of suicide [3], we anticipate this would be a rare occurrence for an individual to report a suicide attempt without suicide ideation. However, this may be possible if participants understand items differently from what was intended by the survey developers.

Finally, there are a number of sub-groups of interest who are not included in our analyses, and for whom the generalizability of our results is unknown. While suicide is among the leading causes of death for people aged 15-29 years [1], in this survey, children and adolescents were excluded entirely (in the case of individuals 14 and younger) or from the child abuse module (in the case of 15-17 year olds). This precluded us from examining more short-term impacts of child abuse. Furthermore, given the reliance on survey data using items assessing respondents' suicide ideation and attempts in the past 12 months, we can speak only to characteristics of those who attempt suicide within 1 year of the onset of suicide ideation, and who survive the suicide attempt(s). Nock and colleagues [3] report that $60 \%$ of individuals who attempt non-lethal suicide do so in the 12 months following onset of suicide ideation. It is unknown whether the same characteristics that we have identified for those with suicide attempts within less than 12 months of the onset of suicide ideation are the same for those whose suicide attempts come at a later point relative to the onset of suicide ideation. Similarly, characteristics may differ between those who die by suicide compared to those who make a non-lethal attempt.

Given the limited number of identified characteristics associated with suicide attempts, our findings are an initial step towards improving identifying the smaller group of individuals who attempt suicide out of the relatively larger group of those reporting thoughts of suicide. They reinforce that factors that may contribute to thoughts of suicide often do not necessarily predict action [2]. While the majority of individuals attempting suicide report child abuse, the association of child abuse, and suicide attempts were attenuated by approximately half when proximal health and social functioning variables, such as the presence of a suicide plan, current mental health, financial situation, and ability to cope with unexpected stressors, were considered. As these proximal factors may be consequences of negative child abuse, taking a life-course perspective on the relationships among these factors is necessary to inform suicide prevention and treatment strategies to reduce initiation and/or persistence of suicide attempts.

\section{Compliance with ethical standards}

Financial and material support The analysis presented in this paper was conducted at the COOL RDC which is part of the Canadian Research Data Centre Network (CRDCN). The services and activities provided by the COOL RDC are made possible by the financial or inkind support of the SSHRC, the CIHR, the CFI, Statistics Canada, Carleton University, the University of Ottawa, and the Universite du Québec en Outaouais. The views expressed in this paper do not necessarily represent the CRDCN's or that of its partners'. Michael Martin acknowledges financial support by a Vanier Canada Graduate Scholarship, and Dr. Colman is supported by the Canada Research Chairs Program.

Role of the sponsors The supporters had no role in the design, analysis, interpretation, or publication of this study.

Conflict of interest None.

\section{References}

1. World Health Organization (2014) Preventing suicide: a global imperative. World Health Organization, Geneva

2. Klonsky ED, May AM (2014) Differentiating suicide attempters from suicide ideators: a critical frontier for suicidology research. Suicide Life Threat Behav 44(1):1-5. doi:10.1111/sltb.12068

3. Nock MK, Borges G, Bromet EJ et al (2008) Cross-national prevalence and risk factors for suicidal ideation, plans and attempts. Br J Psychiatry 192(2):98-105. doi:10.1192/bjp.bp.107. 040113

4. Dube SR, Anda RF, Felitti VJ, Chapman DP, Williamson DF, Giles WH (2001) Childhood abuse, household dysfunction, and the risk of attempted suicide throughout the life span: findings from the Adverse Childhood Experiences Study. J Am Med Assoc 286(24):3089-3096

5. Flannery DJ, Singer MI, Wester K (2001) Violence exposure, psychological trauma, and suicide risk in a community sample of dangerously violent adolescents. J Am Acad Child Adolesc Psychiatry 40(4):435-442. doi:10.1097/00004583-20010400000012

6. Pelkonen M, Marttunen M (2003) Child and adolescent suicide: epidemiology, risk factors, and approaches to prevention. Paediatr Drugs 5(4):243-265

7. Enns MW, Cox BJ, Afifi TO, De Graaf R, Ten Have M, Sareen J (2006) Childhood adversities and risk for suicidal ideation and attempts: a longitudinal population-based study. Psychol Med 36(12):1769-1778. doi:10.1017/S0033291706008646

8. Afifi TO, Enns MW, Cox BJ, Asmundson GJ, Stein MB, Sareen J (2008) Population attributable fractions of psychiatric disorders and suicide ideation and attempts associated with adverse childhood experiences. Am J Public Health 98(5):946-952. doi:10. 2105/AJPH.2007.120253

9. Afifi TO, MacMillan HL, Boyle M, Taillieu T, Cheung K, Sareen J (2014) Child abuse and mental disorders in Canada. Can Med Assoc J 186(9):E324-E332. doi:10.1503/cmaj.131792

10. Maniglio R (2011) The role of child sexual abuse in the etiology of suicide and non-suicidal self-injury. Acta Psychiatr Scand 124(1):30-41. doi:10.1111/j.1600-0447.2010.01612.x

11. O'Connor RC, Nock MK (2014) The psychology of suicidal behaviour. Lancet Psychiatry 1(1):73-85. doi:10.1016/S22150366(14)70222-6

12. Stack S (2014) Differentiating suicide ideators from attempters: violence-a research note. Suicide Life Threat Behav 44(1):46-57. doi:10.1111/sltb.12054

13. McLaughlin KA, Conron KJ, Koenen KC, Gilman SE (2010) Childhood adversity, adult stressful life events, and risk of pastyear psychiatric disorder: a test of the stress sensitization hypothesis in a population-based sample of adults. Psychol Med 40(10):1647-1658. doi:10.1017/S0033291709992121 
14. Joiner TE, Van Orden KA, Witte TK et al (2009) Main predictions of the interpersonal-psychological theory of suicidal behavior: empirical tests in two samples of young adults. J Abnorm Psychol 118(3):634-646. doi:10.1037/a0016500

15. Ribeiro JD, Joiner TE (2011) Present status and future prospects take up the interpersonal-psychological theory of suicidal behaviour. In: O'Connor RC, Platt S, Gordon J (eds) International handbook of suicide prevention: research, policy and practice. Wiley, Chichester, pp 161-179

16. McAuliffe CM (2002) Suicidal ideation as an articulation of intent: a focus for suicide prevention? Arch Suicide Res 6(4):325-338. doi:10.1080/13811110290114106

17. Posner K, Brown GK, Stanley B et al (2011) The ColumbiaSuicide Severity Rating Scale: initial validity and internal consistency findings from three multisite studies with adolescents and adults. Am J Psychiatry 168(12):1266-1277. doi:10.1176/ appi.ajp.2011.10111704

18. Wetzler S, Asnis GM, Bernstein Hyman R, Virtue C, Zimmerman J, Rathus JH (1996) Characteristics of suicidality among adolescents. Suicide Life Threat Behav 26(1):37-45

19. Gunn JF III, Lester D, McSwain S (2011) Testing the warning signs of suicidal behavior among suicide ideators using the 2009 National survey on drug abuse and health. Int J Emerg Ment Health 13(3): 147-154

20. McManama O’Brien KH, Becker SJ, Spirito A, Simon V, Prinstein MJ (2014) Differentiating adolescent suicide attempters from ideators: examining the interaction between depression severity and alcohol use. Suicide Life Threat Behav 44(1):23-33. doi:10.1111/sltb. 12050

21. Pompili M, Innamorati M, Di Vittorio C, Sher L, Girardi P, Amore M (2014) Sociodemographic and clinical differences between suicide ideators and attempters: a study of mood disordered patients 50 years and older. Suicide Life Threat Behav 44(1):34-45. doi:10.1111/sltb.12051

22. Paluszny M, Davenport C, Kim WJ (1991) Suicide attempts and ideation: adolescents evaluated on a pediatric ward. Adolescence 26(101):209-215

23. Wang Y, Sareen J, Afifi TO, Bolton S-L, Johnson EA, Bolton JM (2012) Recent stressful life events and suicide attempt. Psychiatr Ann 42(3):101-108. doi:10.3928/00485713-20120217-07

24. Inder KJ, Handley TE, Johnston A et al (2014) Determinants of suicidal ideation and suicide attempts: parallel cross-sectional analyses examining geographical location. BMC Psychiatry. doi:10.1186/1471-244X-14-208

25. Bazalgette L, Bradley W, Ousbey J (2011) "Hidden data provide new insights into life at the end...": The truth about sucide. Demos, London

26. Webb RT, Kontopantelis E, Doran T, Qin P, Creed F, Kapur N (2012) Suicide risk in primary care patients with major physical diseases. Arch Gen Psychiatry 69(3):256-264
27. Statistics Canada (2013) Canadian Community Health Survey (CCHS) - mental health: user guide. Statistics Canada, Ottawa

28. Walsh CA, MacMillan HL, Trocmé N, Jamieson E, Boyle MH (2008) Measurement of victimization in adolescence: development and validation of the Childhood Experiences of Violence Questionnaire. Child Abuse Negl 32(11):1037-1057. doi:10. 1016/j.chiabu.2008.05.003

29. Kessler RC, Üstün TB (2004) The World Mental Health (WMH) survey initiative version of the World Health Organization (WHO) Composite International Diagnostic Interview (CIDI). Int J Methods Psychiatr Res 13(2):93-121. doi:10.1002/mpr.168

30. Carpiano RM, Hystad PW (2011) "Sense of community belonging" in health surveys: what social capital is it measuring? Health Place 17(2):606-617. doi:10.1016/j.healthplace.2010.12. 018

31. Zou G (2004) A modified poisson regression approach to prospective studies with binary data. Am $\mathrm{J}$ Epidemiol 159(7):702-706. doi:10.1093/aje/kwh090

32. Lopez-Castroman J, Melhem N, Birmaher B et al (2013) Early childhood sexual abuse increases suicidal intent. World Psychiatry 12(2):149-154. doi:10.1002/wps.20039

33. Green JG, Mclaughlin KA, Berglund PA et al (2010) Childhood adversities and adult psychiatric disorders in the National Comorbidity Survey Replication I: associations with first onset of DSM-IV disorders. Arch Gen Psychiatry 67(2):113-123. doi:10. 1001/archgenpsychiatry.2009.186

34. Colman I, Garad Y, Zeng Y et al (2013) Stress and development of depression and heavy drinking in adulthood: moderating effects of childhood trauma. Soc Psychiatry Psychiatr Epidemiol 48(2):265-274. doi:10.1007/s00127-012-0531-8

35. Patten SB (2013) Childhood and adult stressors and major depression risk: interpreting interactions with the sufficientcomponent cause model. Soc Psychiatry Psychiatr Epidemiol 48(6):927-933. doi:10.1007/s00127-012-0603-9

36. Chang S-S, Stuckler D, Yip P, Gunnell D (2013) Impact of 2008 global economic crisis on suicide: time trend study in 54 countries. BMJ 347:f5239. doi:10.1136/bmj.f5239

37. Miller TR, Taylor DM (2005) Adolescent suicidality: who will ideate, who will act? Suicide Life Threat Behav 35(4):425-435

38. O'Connor RC (2011) Towards an integrated motivational-volitional model of suicidal behaviour. In: O'Connor RC, Platt S, Gordon $\mathbf{J}$ (eds) International handbook of suicide prevention: research, policy and practice, 1st edn. Wiley, Chichester, pp 181-198

39. Colman I, Kingsbury M, Garad Y et al (2016) Consistency in adult reporting of adverse childhood experiences. Psychol Med 46(3):543-549. doi:10.1017/S0033291715002032 